\title{
Alternativas de acceso al patrimonio cultural de los ciudadanos europeos
}

\author{
European citizens'alternative modes \\ of engagement with cultural heritage
}

\author{
Victoria Ateca Amestoy* \\ Universidad del País Vasco/Euskal Herriko Unibertsitatea
}

\section{Resumen}

En este artículo caracterizamos los determinantes del acceso de los europeos al patrimonio cultural y analizamos de forma simultánea tres alternativas. El análisis de los datos del Eurobarómetro Especial 466 que se realizó en 2017, pocos meses antes del comienzo del Año Europeo del Patrimonio Cultural, nos permite entender mejor cómo los ciudadanos acceden y disfrutan de elementos del patrimonio cultural a través de diversas manifestaciones y medios. En concreto, nos centramos en el análisis de las visitas a monumentos e instituciones patrimoniales, en la participación en manifestaciones de patrimonio inmaterial y en el acceso digital. Encontramos evidencia de un alto grado de complementariedad entre las alternativas.

Palabras clave: participación, patrimonio cultural, patrimonio digital, patrimonio material e inmaterial, Unión Europea, Eurobarómetro Especial 466.

Clasificación JEL: Z1O.

\begin{abstract}
We characterize the determinants of European citizens' participation in cultural heritage by simultaneously analyzing three alternative ways of engagement. By using the data from the Special Eurobarometer 466 from 2017, a survey undertaken some months before European Year of Cultural Heritage started, we can better understand how citizens access and enjoy the elements of cultural heritage through different modes of participation. We analyze visits to monuments and heritage institutions, the participation in intangible cultural heritage expressions, and digital access. We find supporting evidence for relevant complementarities among them.
\end{abstract}

Keywords: participation, cultural heritage, digital heritage, material and intangible heritage, European Union, Special Eurobarometer 466.

JEL classification: Z10.

* Este trabajo se enmarca en los proyectos de investigación sobre bienes culturales y mercados que se desarrollan en la Universidad del País Vasco/ Euskal Herriko Unibertsitatea y que están financiados por el Ministerio de Ciencia, Innovación y Universidades (ECO2015-64467-R) y por el Gobierno Vasco (IT336-19). La investigación se enriqueció gracias a los comentarios recibidos en el marco del proyecto «PROtect traditional built HERITAGE Skills-PRO-Heritage» (H2020-EE-2017-CSA-PPI; Grant agreement ID: 785211). 


\section{Introducción}

La aproximación económica al estudio del comportamiento humano se puede aplicar al análisis de cómo y por qué los ciudadanos deciden relacionarse con el patrimonio cultural. Existen múltiples razones por las que los individuos acceden de forma física o inmaterial a los elementos heredados del pasado que contienen símbolos relevantes para su comunidad o para la identidad de otro grupo. Los valores imbuidos en elementos materiales e inmateriales heredados del pasado (a veces de un pasado reciente) están interpretados desde el presente y son las generaciones actuales las que deciden qué merece ser preservado y transmitido a generaciones futuras. En este artículo nos centramos en un aspecto relevante de las decisiones de las generaciones presentes: cómo disfrutar del patrimonio cultural y acceder a él.

El concepto propio de patrimonio cultural ha sufrido cambios a lo largo del tiempo (Vecco, 2010; Benhamou, 2011; Ateca-Amestoy, 2013). Podemos destacar varios factores que han provocado los cambios que han desembocado en la extensión del concepto. En primer lugar, se extendió al incluir manifestaciones del patrimonio inmaterial. En segundo lugar, se acogió a nuevas categorías, como paisaje cultural, en un proceso de reconocimiento de que los elementos singulares son solo relevantes dentro de su contexto geográfico y en relación a sus comunidades. En tercer lugar, se consideró que tanto el patrimonio nacido digital como el que se ha digitalizado son vestigios y formas de conservar el pasado.

El acceso al patrimonio es condición necesaria para su valoración e incluso, en muchos casos de manifestaciones de patrimonio inmaterial, para su existencia misma. La comprensión y el disfrute de la dimensión simbólica de los bienes patrimoniales son necesarios para que los ciudadanos accedan y conserven esos elementos. La participación en el patrimonio cultural puede tomar múltiples formas, pero el acceso, la conservación y el disfrute han de estar presentes en cualquiera de ellas. En el caso del patrimonio intangible y de sus manifestaciones, es crucial reconocer que las herramientas que garantizan su conservación y su transmisión son el acceso de las llamadas comunidades de práctica, que a través de su participación, mantienen esas tradiciones vivas y las recrean y transmiten.

Uno de los dilemas tradicionales en el área del patrimonio ha sido la imposibilidad de garantizar la conservación de los elementos y el acceso de los ciudadanos al mismo tiempo. Muchos de los acuerdos tradicionales y las instituciones del patrimonio asignaban una función de vigilantes a los expertos, que determinaban qué merecía la pena de ser conservado y cuál era la mejor manera de hacerlo. Poco a poco, este modelo ha ido cambiando, reconociendo el papel central que las comunidades tienen en la conservación, así como su derecho de acceso. En cierta medida, cambios tecnológicos como la digitalización han ayudado a superar este dilema facilitando, por ejemplo, instrumentos de gestión que podrían solventar los problemas de congestión que experimentan algunos recursos frágiles y sobreexplotados.

En este artículo vamos a aplicar el análisis económico de la participación cultural para comprender qué determina que los ciudadanos europeos accedan al patrimonio 
material, al inmaterial y de forma tanto presencial como virtual. La aproximación económica al comportamiento humano es una herramienta válida para examinar cuestiones como los determinantes del acceso, las diferencias por grupos sociales y las posibles complementariedades o efectos de sustitución entre unas formas y otras.

\section{Acceso y participación en el patrimonio cultural}

La decisión de acceso al patrimonio cultural depende de diferentes factores. En general, los individuos han de decidir cómo asignar de forma óptima recursos escasos (sobre todo su tiempo y dinero) para satisfacer una serie de necesidades mediante la producción y disfrute de lo que podemos llamar «apreciación cultural» siguiendo a Becker (1965). Para comprender el concepto de patrimonio cultural y participación, podemos acudir a la versión de 2009 del Marco de Estadísticas Culturales de la UNESCO (UNESCO, 2009), en el que se definen las fases del «ciclo cultural», una aproximación parecida a la cadena de valor (Comisión Europea, 2017a), que explora la interacción entre diferentes etapas que van de la creación artística a la participación. La participación es la última etapa y es tan importante como el acto creativo; si los ciudadanos no llegan a poder disfrutar y conocer los bienes del patrimonio, no se puede cerrar el ciclo cultural. Los bienes del patrimonio quedan caracterizados dentro del Marco como poseedores de valores simbólicos, históricos, artísticos, estéticos, etnológicos o antropológicos, científicos o sociales. En cualquier caso, se trata de dimensiones del llamado «valor cultural» (Throsby, 2001) y son inherentes a los bienes del patrimonio cultural, sean materiales, inmateriales o digitales.

Desde el punto de vista económico, cuando consideramos las propiedades de los bienes que pertenecen al patrimonio cultural, tenemos que tener en cuenta sus características de bien público, las externalidades derivadas del consumo, los aspectos de sostenibilidad del capital cultural y los procesos de puesta en valor del patrimonio cultural para conseguir actividades que generen actividad económica (medida, sobre todo, en términos de ingresos fiscales y de empleo). Algunas presentaciones de la configuración económica del patrimonio cultural y sus implicaciones se pueden encontrar en Greffe (1989), Peacock (1995 y 1998), Throsby (1997), Peacock y Rizzo (2008), Benhamou (2011) y Ateca-Amestoy (2013).

Para las tres formas de patrimonio que consideramos en este artículo, las características especiales y definitorias de los bienes culturales como bienes económicos son perfectamente aplicables. También lo es la aproximación económica al estudio de la participación cultural: los individuos han de combinar recursos personales escasos para satisfacer sus necesidades culturales. En este artículo pretendemos explorar las posibles diferencias entre variables que explican el acceso al patrimonio cultural de los ciudadanos de Europa.

Es posible que el precio implícito final al que se enfrenta cada ciudadano a la hora de acceder de una u otra forma al patrimonio cultural sea muy diferente, aunque el precio de taquilla sea el mismo (por ejemplo, cero). Mientras que la participación a 
través de visitas exige desplazarse hasta el museo o monumento, dedicar un tiempo a llegar físicamente hasta allí y coincidir con el horario de apertura, la participación a través de algunas manifestaciones del patrimonio inmaterial requiere una alta especialización (como la práctica de oficios tradicionales), o dedicación de tiempo (como la participación en celebraciones comunitarias). En el caso de la participación digital, la especialización pasará por disponer del equipamiento y de las habilidades digitales para acceder a contenidos. Otras diferencias también se pueden deber a los factores de contexto que explican la disponibilidad de acceso. En algunos casos, están relacionados con factores de oferta y economías de aglomeración; en otros, con infraestructura de servicios y comunicaciones. Habrá también unos factores comunes que expliquen que, siendo bienes altamente simbólicos, su disfrute ha de estar influido por el acervo cultural del individuo, adquirido a través de su educación formal o a través de procesos informales de socialización y de pertenencia en la comunidad.

Para justificar nuestro análisis, pasamos a presentar un repaso de la evidencia sobre los determinantes de la participación en esas tres alternativas. En este artículo no consideramos si las actividades pertenecen o no a la llamada «alta cultura» como en los análisis de la distinción motivados en los estudios de Bourdieu (1979), o en los análisis de «omnívoros» o «unívoros», como en Peterson y Kern (1996). En nuestro caso, es más interesante el análisis de Novak-Leonard y Brown (2011) sobre el concepto multimodal de participación cultural, que va mucho más allá de la asistencia a espectáculos en vivo y la visita a instituciones culturales.

Para el patrimonio material, existe amplia evidencia sobre el papel de la educación como principal determinante del interés o de la falta de interés que puede operar como barrera a la participación a través de visitas a monumentos o museos: la educación determina el capital cultural que tiene el individuo. Es fundamental en los modelos que explican diferencias en la participación cultural (Becker, 1965; Sable y Kling, 2001; Lévy-Garboua y Montmarquette, 2011) y es, sistemáticamente, el mejor predictor de asistencia (Ateca-Amestoy y Prieto-Rodríguez, 2013). Otros trabajos empíricos buscan resultados en términos de sexo (identificando una brecha a favor de las mujeres), edad, composición del hogar, ocupación... (Seaman, 2005). El artículo de Falk y Katz-Gerro (2016) en el que analizan la frecuencia de visitas a sitios de interés cultural por los europeos utilizando datos de la Encuesta Europea de Ingresos y Condiciones de Vida (EU-SILC) del año 2006 ofrece una visión completa de los determinantes de la intensidad del acceso al patrimonio. Uno de los resultados más interesantes que obtienen es la persistencia de la educación como factor de acceso en diferentes países. Para el caso español, Suárez-Fernández et al. (2019) utilizan los datos para España de la edición de esa misma encuesta de 2015 y realizan un análisis comparativo del efecto de la renta y de la educación sobre la intensidad de la participación en patrimonio, cine y espectáculos en vivo.

El acceso al patrimonio material está, en muchos casos, relacionado con el auge del turismo cultural. Se trata de una categoría difícil de delimitar, existiendo un debate vivo sobre la necesidad de definirlo en términos de la motivación del viajero o de 
su comportamiento en destino (Bonet, 2013; Guccio et al., 2017). La Organización Mundial de Turismo (UNWTO) parece favorecer el punto de vista de las motivaciones, donde la motivación cultural estaría relacionada con el aprendizaje, el descubrimiento, la experimentación y el consumo del patrimonio (UNWTO, 2018). Los economistas de la cultura prestan una atención renovada al fenómeno del turismo cultural (Noonan y Rizzo, 2017), al existir evidencia de la importancia de ese segmento dentro de los públicos de las instituciones culturales, que han de acomodarse a la coexistencia de público ocasional de instituciones culturales (solo cuando viajan, como encuentran Brida et al., 2016) y de público fuertemente motivado (Guccio et al., 2017).

En este trabajo se presentan las categorías de patrimonio material e inmaterial como separadas cuando, en la realidad, coexisten en muchas ocasiones. Como en el caso del paisaje cultural, muchos destinos culturales y muchos bienes patrimoniales tienen características «mixtas» y existe una fuerte complementariedad entre los aspectos materiales -edificación-e inmateriales -manifestaciones y tradiciones históricas-, por ejemplo (Guccio et al., 2017).

Hay pocos trabajos en la literatura económica que analicen el acceso al patrimonio intangible. Ateca-Amestoy et al. (2019) analizan la intensidad de la participación en patrimonio material e inmaterial (para la manifestación de celebraciones históricas y comunitarias) en Iberoamérica y encuentran algunas diferencias en los factores que operan como barrera y como impulsor de la participación. Para el caso del patrimonio inmaterial, variables relacionadas con participación y capital social tienen un papel relevante. Es un resultado en línea con los hallazgos de Palma et al. (2013) sobre la importancia de las variables que representan vínculos personales para explicar la participación en las Fiestas de Primavera en Sevilla (Semana Santa, Feria y toros), que queda caracterizado como un bien cultural complejo (mixto) que la comunidad produce con la combinación de patrimonio material (inmueble y mueble) e inmaterial.

El análisis de los determinantes de la participación en patrimonio cultural comienza a tener en cuenta también las nuevas posibilidades de acceso digital y la consolidación de una nueva categoría, la de patrimonio digital, con especiales características. La Unión Europea reconoce la categoría del patrimonio digital, sean elementos digitalizados o elementos ya nacidos digitales, como un conjunto de bienes que reúnen los valores definitorios del patrimonio cultural. El proceso de digitalización ha creado nuevas instituciones culturales, como los museos virtuales, planteando importantes retos a instituciones como el Consejo Internacional de Museos (ICOM) en la propia redefinición de museo (Navarrete, 2013). También ha revolucionado funciones y procedimientos en instituciones como archivos y bibliotecas (Guccio et al., 2016). Ha cambiado los modelos de negocio del sector del patrimonio y dentro de los elementos de estos, sobre todo, el desarrollo de audiencias y las cadenas de valor, con una menor incidencia en los procesos de monetización (Comisión Europea, 2017a). En lo que respecta a las audiencias, la digitalización ha transformado la demanda de patrimonio de diferentes formas, ya que ha ayudado a superar el dilema 
tradicional entre garantizar la conservación o el acceso a los bienes culturales (Rizzo y Towse, 2016). Así, la relación digital ha posibilitado más oportunidades de relaciones más profundas con las audiencias animando, por ejemplo, a las visitas repetidas y más activas de aquellos ciudadanos que ya participaban. También ha posibilitado la ampliación de las audiencias gracias a la interactividad, conectividad y convergencia del acceso digital. Sin embargo, no existe evidencia concluyente sobre cómo esta última posibilidad se ha podido convertir en un acceso efectivo mayor. Para un estudio de la relación entre acceso físico y digital en artes escénicas en España, De la Vega et al. (2019) encuentran que la probabilidad de acceso exclusivo online es mayor para los individuos que declaran que el precio y la falta de oferta son las variables que limitan su participación en vivo. Las nuevas generaciones (aquellos que son nativos digitales) son más competentes digitalmente y tienen, en general, unas preferencias marcadas por los usos sociales de la tecnología, lo que abre también la puerta a que los grupos más jóvenes sean audiencias puramente virtuales (Evrand y Krebs, 2018).

Algunas investigaciones en el área de museos y patrimonio parecen apoyar que las experiencias digitales son complementarias y no sustitutivas de las visitas y formas de acceder tradicionales (Evrand y Krebs, 2018; Ateca-Amestoy y Castiglione, 2014; Mihelj et al., 2019). Los medios digitales podrían atraer a nuevas audiencias a los museos y a las instituciones del patrimonio, pero el acceso sigue siendo muy desigual y relacionado con diferentes niveles educativos y clase social (Reeves, 2019). Sin embargo, estos estudios también parecen indicar que la brecha digital de segundo orden, que es la que explica que diferentes grupos sociales accedan a internet para llevar a cabo diferentes actividades, también podría exacerbar las tradicionales divisiones en el acceso (para una comparación entre diferentes formas culturales en Estados Unidos, ver Ateca-Amestoy y Castiglione, 2016). Con los mismos datos que analizamos en este artículo, en Ateca-Amestoy (2018) se realiza un análisis que encuentra evidencia a favor de las complementariedades entre esas formas de acceso.

\section{Acceso multimodal en Europa}

Hasta hace poco era difícil conocer las tasas de participación de los ciudadanos europeos en el patrimonio cultural. La mayor parte de las estadísticas comparables a nivel europeo se han centrado en los monumentos y en instituciones como los museos. Uno de los estudios más completos hasta la fecha es el análisis de Falk y Katz-Gerro (2016) en el que analizan la frecuencia de visitas a sitios de interés cultural por los europeos utilizando datos de la Encuesta Europea de Ingresos y Condiciones de Vida (EU-SILC) del año 2006. Algunos estudios, como Lagaert y Roose (2018) utilizaron datos del Eurobarómetro para conocer la brecha de género en la participación cultural, concluyendo que existen diferencias notables entre países, ya que la dimensión de género relacionada con la participación cultural se determina por factores micro (individuales), meso (de contexto) y macro (de políticas y otras variables nacionales). 
En 2018 se celebró el Año Europeo del Patrimonio Cultural y, como acción preparatoria, la Unión Europea decidió impulsar un Eurobarómetro Especial que se dedicó en gran parte a conocer mejor cómo valoran los ciudadanos europeos el patrimonio cultural y cómo acceden a él. Se midieron percepciones, actitudes y comportamientos de los ciudadanos en relación al patrimonio cultural. El cuestionario fue especialmente rico en la consideración de un amplio concepto de patrimonio, diferenciando entre la dimensión material, la inmaterial y el patrimonio digital, aunque esta última dimensión se centra sobre todo en el acceso digital al patrimonio material o inmaterial. El Eurobarómetro Especial 466 (incluido en Eurobarómetro 88.1) recogió la información individual de una muestra de 27.881 sujetos en los 28 países de la Unión Europea (Comisión Europea, 2017b y 2017c).

El cuestionario sobre patrimonio comienza con el siguiente texto sobre la definición del patrimonio:

Patrimonio cultural es un término que se utiliza para identificar una serie de recursos que se protegen, conservan o recuperan porque se considera que deben transmitirse a las generaciones futuras. El patrimonio cultural puede hacer referencia a objetos concretos, como monumentos, yacimientos arqueológicos, obras de arte, películas, libros o documentos conservados y administrados por museos, bibliotecas y archivos; son también cosas intangibles, como habilidades, rituales, música y actos festivos. Actualmente, el patrimonio cultural también puede ser digital, como arte digital o reproducciones digitales de patrimonio cultural. El patrimonio cultural de Europa hace referencia al patrimonio cultural de Europa y de los países que forman parte de esta, considerado de forma individual o colectiva.

Con la información disponible en la encuesta podemos explicar la decisión de participación a través de diferentes modos. En primer lugar, consideramos la participación en la dimensión tangible de patrimonio inmueble; en segundo lugar, la participación en la dimensión inmaterial; por último, el acceso digital antes, durante o después de la participación en el patrimonio material o inmaterial. El Cuadro 1 presenta los elementos de la encuesta que hemos utilizado para cada una de esas dimensiones junto con el literal de la pregunta del cuestionario y el porcentaje estimado de la población europea que ha realizado o realiza esa actividad. Las tasas de participación varían entre el $60,92 \%$ para las visitas a monumentos y sitios históricos, la actividad más popular y el 7,61\% que corresponde a la posesión de habilidades y conocimientos para realizar artesanía tradicional, una actividad mucho más demandante en términos del capital humano específico que tiene que tener el individuo para llevarla a cabo. Sobre el acceso digital, parece que el interés o la necesidad de información de los individuos es mayor antes de la visita que durante o después. 


\section{CUADRO 1 \\ MODOS DE ACCESO Y PARTICIPACIÓN RELACIONADOS CON EL PATRIMONIO CULTURAL EN EUROPA}

\begin{tabular}{|c|c|c|}
\hline $\begin{array}{l}\text { Material } \\
\text { Durante los últimos } 12 \text { meses... }\end{array}$ & & $\begin{array}{l}\text { Proporción } \\
(\%)\end{array}$ \\
\hline museos & ¿Ha visitado un museo o galería de arte? & 49,51 \\
\hline monumentos & $\begin{array}{l}\text { ¿Ha visitado un monumento } \\
\text { o lugar histórico (palacios, castillos, iglesias, yacimientos } \\
\text { arqueológicos, jardines, etc.)? }\end{array}$ & 60,92 \\
\hline bibliotecas y archivos & $\begin{array}{l}\text { ¿Ha visitado una biblioteca o archivo (p. ej., para consultar } \\
\text { manuscritos, documentos, mapas antiguos, etc.)? }\end{array}$ & 29,11 \\
\hline \multicolumn{3}{|l|}{$\begin{array}{l}\text { Inmaterial } \\
\text { Durante los últimos } 12 \text { meses... } \\
\text { ¿Está usted involucrado/a de } \\
\text { alguna forma en el ámbito del } \\
\text { patrimonio cultural? }\end{array}$} \\
\hline artes escénicas & $\begin{array}{l}\text { ¿Ha asistido a un evento de artes escénicas tradicionales o } \\
\text { clásicas (p. ej., música, incluyendo ópera, danza o teatro, } \\
\text { música popular, etc.)? }\end{array}$ & 42,32 \\
\hline artesanía & $\begin{array}{l}\text { ¿Ha visitado un taller de artesanía tradicional (p. ej., tejido, } \\
\text { soplado de vidrio, arte decorativo, bordados, fabricación de } \\
\text { instrumentos musicales, cerámica, etc.)? }\end{array}$ & 29,86 \\
\hline eventos tradicionales & $\begin{array}{l}\text { ¿Ha asistido a un evento tradicional (p. ej., festival } \\
\text { gastronómico, carnaval, teatro de marionetas, festival de } \\
\text { flores, etc.)? }\end{array}$ & 51,82 \\
\hline actividad tradicional & $\begin{array}{l}\text { Realiza una actividad tradicional, como baile o canto } \\
\text { tradicional, toca música tradicional, cocina tradicional, etc. }\end{array}$ & 8,11 \\
\hline artesanía & $\begin{array}{l}\text { Posee las habilidades o los conocimientos para realizar uno } \\
\text { o más tipos de artesanía tradicional (p. ej., tejido, arte de- } \\
\text { corativo, bordados, fabricación de instrumentos musicales, } \\
\text { cerámica, etc) }\end{array}$ & 7,61 \\
\hline cine europeo clásico & $\begin{array}{l}\text { ¿Ha ido al cine o a un festival de patrimonio cinematográfico } \\
\text { para ver un clásico de la cinematografía europea producido } \\
\text { hace como mínimo } 10 \text { años? }\end{array}$ & 25,79 \\
\hline \multirow[t]{4}{*}{$\begin{array}{r}\text { Digital } \\
\text { ¿Ha usado internet durante } \\
\text { los últimos } 12 \text { meses para los } \\
\text { siguientes fines relacionados con } \\
\text { el patrimonio cultural? }\end{array}$} & & \\
\hline & $\begin{array}{l}\text { Buscar información general relacionada con el patrimonio } \\
\text { cultural, como accesibilidad, instalaciones y principales } \\
\text { características de un museo, monumento histórico o evento } \\
\text { tradicional al preparar una visita o unas vacaciones. }\end{array}$ & 31,34 \\
\hline & $\begin{array}{l}\text { Consultar contenido relacionado con el patrimonio cultural, } \\
\text { como la descripción de una obra de arte o un monumento } \\
\text { histórico durante una visita, información histórica sobre un } \\
\text { evento tradicional al cual asiste, etc. }\end{array}$ & 20,57 \\
\hline & $\begin{array}{l}\text { Obtener más información sobre un museo o una fiesta } \\
\text { tradicional, monumentos históricos, o exposición después } \\
\text { de una visita. }\end{array}$ & 19,13 \\
\hline
\end{tabular}

FUENTE: Elaboración propia basada en datos del Eurobarómetro Especial 466 (2017). 


\section{Métodos y especificación empírica}

Como queremos aprovechar la información derivada de observar de forma simultánea la participación a través de las tres manifestaciones que hemos descrito, estimamos un modelo probit multivariante, tal y como definen Cappellari y Jenkins (2003), que es estimado por máxima verosimilitud simulada. Se trata de una extensión de los modelos probit bivariados que permite considerar la correlación entre los términos de error aleatorio de cada una de las ecuaciones que componen el sistema (en nuestro caso, 3). Una de las ventajas de estimar este modelo es que nos permite considerar los factores que influyen en cada una de las decisiones de participación y, al mismo tiempo, las posibles relaciones entre las diferentes formas de participación. Como señalan Cheng y Wen (2011) en su análisis de la asistencia a diferentes manifestaciones de artes escénicas y musicales en Taiwan, el método facilita considerar el solapamiento que se produce entre las audiencias de diferentes manifestaciones culturales, un fenómeno muy frecuente tal y como señala también Seaman (2005).

Para llevar a cabo nuestro análisis consideramos de forma simultánea las tres formas de acceso al patrimonio cultural que hemos mencionado: patrimonio tangible e instituciones, patrimonio inmaterial y acceso digital al patrimonio o patrimonio digital. Así, se estima un modelo con tres ecuaciones con las mismas variables explicativas: características demográficas de los individuos (sexo, ser o no nacional del país donde reside, edad, estado civil y presencia de niños en el hogar), características socio-económicas (educación, situación laboral, posición política, dificultades económicas y clase social declarada) y localización (país). Además, como individuos que viven en la misma región pueden acceder a una oferta común de elementos patrimoniales, modelamos los errores estándares para que estén clusterizados por región (al nivel de desagregación NUTS2). Los estadísticos descriptivos de la muestra para las variables dependientes y explicativas están recogidos en el Cuadro 2.

\section{CUADRO 2}

ESTADÍSTICOS DESCRIPTIVOS DE LA MUESTRA

\begin{tabular}{|l|c|}
\hline & Proporción (\%) \\
\hline Variables dependientes & \\
\hline Patrimonio material & 68,48 \\
\hline Patrimonio inmaterial & 69,78 \\
\hline Acceso digital/patrimonio digital & 41,45 \\
\hline Variables explicativas & \\
\hline Sexo & \\
\hline Hombre & 44,82 \\
\hline
\end{tabular}


CUADRO 2 (Continuación)

ESTADÍSTICOS DESCRIPTIVOS DE LA MUESTRA

\begin{tabular}{|c|c|}
\hline & Proporción (\%) \\
\hline \multicolumn{2}{|l|}{ Nacionalidad } \\
\hline Extranjero & 9,95 \\
\hline \multicolumn{2}{|l|}{ Edad } \\
\hline 15-24 años & 8,42 \\
\hline 25-34 años & 12,75 \\
\hline 35-44 años & 15,77 \\
\hline 45-54 años & 16,34 \\
\hline 55-64 años & 18,15 \\
\hline 65-74 años & 17,36 \\
\hline $75+$ & 11,20 \\
\hline \multicolumn{2}{|l|}{ Política izquierda-derecha } \\
\hline $\operatorname{lr} 1$ & 8,05 \\
\hline $\operatorname{lr} 2$ & 16,40 \\
\hline $\operatorname{lr} 3$ & 35,27 \\
\hline $\operatorname{lr} 4$ & 14,67 \\
\hline $\operatorname{lr} 5$ & 6,86 \\
\hline \multicolumn{2}{|l|}{ Estado civil } \\
\hline Casado & 53,03 \\
\hline Soltero con pareja & 11,64 \\
\hline Soltero & 16,28 \\
\hline Divorciado/separado & 7,68 \\
\hline Viudo & 10,65 \\
\hline \multicolumn{2}{|l|}{ Niños en el hogar } \\
\hline Sí & 35,45 \\
\hline \multicolumn{2}{|l|}{ Años de educación } \\
\hline Sin educación formal & 0,77 \\
\hline Educación $<15$ años & 14,15 \\
\hline Nivel de educación ( 16-19 años) & 43,35 \\
\hline Educación 20 años o más & 34,17 \\
\hline Todavía estudiando & 5,72 \\
\hline
\end{tabular}




\section{CUADRO 2 (Continuación) ESTADÍSTICOS DESCRIPTIVOS DE LA MUESTRA}

\begin{tabular}{|l|c|}
\hline & Proporción (\%) \\
\hline Situación laboral & 7,03 \\
\hline Autónomos & 11,06 \\
\hline Gestores & 12,30 \\
\hline Otros profesionales & 20,28 \\
\hline Trabajadores manuales & 43,60 \\
\hline No activos (estudiantes/jubilados/hogar) & 5,74 \\
\hline Parados & \\
\hline Dificultades económicas & 9,39 \\
\hline Dificultades económicas muy frecuentes & 25,05 \\
\hline Dificultades económicas ocasionales & 63,74 \\
\hline Dificultades económicas nunca o casi nunca & \\
\hline Clase social reportada & 27,36 \\
\hline Clase trabajadora & 14,72 \\
\hline Clase media baja & 45,98 \\
\hline Clase media & 6,86 \\
\hline Clase media alta & 0,72 \\
\hline Clase alta & 33,14 \\
\hline Tamaño de comunidad & 36,97 \\
\hline Área rural & \\
\hline Ciudades/áreas urbanas pequeñas & \\
\hline Ciudades y áreas urbanas grandes & \\
\hline
\end{tabular}

NOTA: Algunas categorías no suman el $100 \%$ de la muestra al no considerarse respuestas como «no sabe»o «no contesta».

FUENTE: Elaboración propia basada en datos del Eurobarómetro Especial 466 (2017).

\section{Resultados}

El Cuadro 3 presenta los resultados de la estimación del modelo probit multivariante para las tres formas de acceso que consideramos en este artículo. Como esperábamos, los valores de los coeficientes de correlación estimados son positivos y estadísticamente significativos. Esto puede ser interpretado como una medida de la complementariedad que las tres alternativas presentan entre sí y, en línea con Cheng y Wen (2011), como evidencia a favor del omnivorismo de la participación cultural en el área del patrimonio. El mayor valor del coeficiente de correlación estimado es para la relación entre el acceso al patrimonio inmueble y el acceso digital $(0,5837)$, seguido del de patrimonio inmueble e inmaterial $(0,4457)$ y del de patrimonio inmaterial y acceso digital $(0,3284)$. 


\section{CUADRO 3 \\ RESULTADOS DE LA ESTIMACIÓN DEL MODELO PROBIT MULTIVARIANTE}

\begin{tabular}{|c|c|c|c|c|c|c|}
\hline & \multicolumn{2}{|c|}{ Material } & \multicolumn{2}{|c|}{ Inmaterial } & \multicolumn{2}{|c|}{ Digital } \\
\hline & $\begin{array}{l}\text { Coefi- } \\
\text { ciente }\end{array}$ & $P>|z|$ & $\begin{array}{l}\text { Coefi- } \\
\text { ciente }\end{array}$ & $P>|z|$ & $\begin{array}{l}\text { Coefi- } \\
\text { ciente }\end{array}$ & $P>|z|$ \\
\hline \multicolumn{7}{|l|}{ Sexo } \\
\hline Hombre & $-0,0833$ & 0,0080 & $-0,2159$ & 0,0000 & 0,0004 & 0,9870 \\
\hline \multicolumn{7}{|c|}{ Nacional del país donde reside } \\
\hline Extranjero & $-0,1408$ & 0,0760 & 0,0059 & 0,9670 & $-0,1275$ & 0,1110 \\
\hline \multicolumn{7}{|l|}{ Edad (45-54 años) } \\
\hline 15-24 años & 0,2214 & 0,0040 & $-0,0349$ & 0,7370 & $-0,1111$ & 0,1730 \\
\hline 25-34 años & 0,0853 & 0,1560 & 0,0540 & 0,4430 & 0,0336 & 0,5260 \\
\hline 35-44 años & 0,1206 & 0,0240 & $-0,0107$ & 0,8460 & $-0,0179$ & 0,6880 \\
\hline 55-64 años & 0,0836 & 0,1090 & 0,1824 & 0,0100 & 0,0249 & 0,6010 \\
\hline 65-74 años & 0,1459 & 0,0300 & 0,1657 & 0,0340 & $-0,1919$ & 0,0090 \\
\hline $75+$ & $-0,1934$ & 0,0150 & $-0,0312$ & 0,7450 & $-0,6031$ & 0,0000 \\
\hline \multicolumn{7}{|c|}{ Política izquierda-derecha en 5 puntos (Ir3) } \\
\hline $\operatorname{lr} 1$ & 0,2547 & 0,0000 & 0,2865 & 0,0000 & 0,2502 & 0,0000 \\
\hline $\operatorname{lr} 2$ & 0,2488 & 0,0000 & 0,2222 & 0,0000 & 0,2710 & 0,0000 \\
\hline $\operatorname{lr} 4$ & 0,0849 & 0,1730 & 0,0799 & 0,1930 & 0,1471 & 0,0000 \\
\hline $\operatorname{lr} 5$ & 0,0329 & 0,6170 & 0,1648 & 0,1620 & 0,1360 & 0,0240 \\
\hline \multicolumn{7}{|l|}{ Estado civil (casado) } \\
\hline Soltero con pareja & 0,0299 & 0,6100 & 0,0831 & 0,2800 & 0,0430 & 0,3850 \\
\hline Soltero & $-0,1324$ & 0,0070 & 0,0239 & 0,7220 & $-0,0698$ & 0,1840 \\
\hline Divorciado/separado & $-0,0098$ & 0,8710 & $-0,0482$ & 0,5290 & $-0,0694$ & 0,2050 \\
\hline Viudo & $-0,1481$ & 0,0160 & $-0,0603$ & 0,4210 & $-0,1867$ & 0,0000 \\
\hline \multicolumn{7}{|l|}{ Niños en el hogar } \\
\hline Sí & 0,0090 & 0,8270 & $-0,0367$ & 0,4150 & 0,0135 & 0,7080 \\
\hline \multicolumn{7}{|c|}{ Nivel de educación (sin educación formal) } \\
\hline Educación $<15$ años & 0,3463 & 0,0090 & $-0,0842$ & 0,6900 & 0,3195 & 0,0960 \\
\hline Educación 16-19 años & 0,7987 & 0,0000 & 0,0217 & 0,9160 & 0,6091 & 0,0010 \\
\hline Educación20añosomás & 1,2320 & 0,0000 & 0,2261 & 0,2710 & 1,0509 & 0,0000 \\
\hline Todavía estudiando & 1,3752 & 0,0000 & 0,2483 & 0,3320 & 1,1941 & 0,0000 \\
\hline
\end{tabular}




\section{CUADRO 3 (Continuación)}

\section{RESULTADOS DE LA ESTIMACIÓN DEL MODELO PROBIT MULTIVARIANTE}

\begin{tabular}{|c|c|c|c|c|c|c|}
\hline & \multicolumn{2}{|c|}{ Material } & \multicolumn{2}{|c|}{ Inmaterial } & \multicolumn{2}{|c|}{ Digital } \\
\hline & $\begin{array}{l}\text { Coefi- } \\
\text { ciente }\end{array}$ & $\mathbf{P}>|\mathbf{z}|$ & $\begin{array}{l}\text { Coefi- } \\
\text { ciente }\end{array}$ & $\mathbf{P}>|\mathbf{z}|$ & $\begin{array}{l}\text { Coefi- } \\
\text { ciente }\end{array}$ & $\mathbf{P}>|\mathbf{z}|$ \\
\hline \multicolumn{7}{|c|}{ Situación laboral (trabajadores manuales) } \\
\hline Autónomos & 0,0752 & 0,2350 & 0,1362 & 0,1490 & 0,1316 & 0,0270 \\
\hline Gestores & 0,4346 & 0,0000 & $-0,0246$ & 0,8060 & 0,3630 & 0,0000 \\
\hline Otros profesionales & 0,2109 & 0,0000 & $-0,1576$ & 0,0470 & 0,1517 & 0,0030 \\
\hline $\begin{array}{l}\text { No activos (estudiantes/ } \\
\text { jubilados/hogar) }\end{array}$ & $-0,0969$ & 0,0330 & $-0,1085$ & 0,1330 & $-0,0447$ & 0,3770 \\
\hline Parados & $-0,0786$ & 0,2770 & $-0,0269$ & 0,7750 & $-0,1392$ & 0,0620 \\
\hline \multicolumn{7}{|c|}{ Dificultades económicas (nunca o casi nunca) } \\
\hline $\begin{array}{l}\text { Dificultades económicas } \\
\text { muy frecuentes }\end{array}$ & $-0,3221$ & 0,0000 & 0,1319 & 0,1240 & $-0,0683$ & 0,2840 \\
\hline $\begin{array}{l}\text { Dificultades económicas } \\
\text { ocasionales }\end{array}$ & $-0,0859$ & 0,0860 & 0,0513 & 0,2950 & 0,0065 & 0,8650 \\
\hline \multicolumn{7}{|c|}{ Clase social reportada (clase media) } \\
\hline Clase trabajadora & $-0,3350$ & 0,0000 & $-0,2021$ & 0,0010 & $-0,3084$ & 0,0000 \\
\hline Clase media baja & $-0,0910$ & 0,1140 & $-0,0866$ & 0,0640 & $-0,1202$ & 0,0090 \\
\hline Clase media alta & 0,2601 & 0,0000 & 0,0209 & 0,7350 & 0,2596 & 0,0000 \\
\hline Clase alta & 0,3976 & 0,0780 & $-0,2079$ & 0,3030 & 0,1453 & 0,2940 \\
\hline \multicolumn{7}{|c|}{ Tamaño de comunidad (área rural) } \\
\hline $\begin{array}{l}\text { Ciudades/áreas urbanas } \\
\text { pequeñas }\end{array}$ & 0,0532 & 0,3040 & 0,0855 & 0,1270 & 0,1244 & 0,0200 \\
\hline $\begin{array}{l}\text { Ciudades y áreas urba- } \\
\text { nas grandes }\end{array}$ & 0,1093 & 0,0450 & 0,0980 & 0,0540 & 0,1396 & 0,0060 \\
\hline \multicolumn{7}{|l|}{ País (España) } \\
\hline Bélgica & $-0,0645$ & 0,5940 & 0,1162 & 0,3730 & 0,3608 & 0,0000 \\
\hline Dinamarca & 0,4347 & 0,0000 & 0,4121 & 0,0020 & 0,4993 & 0,0000 \\
\hline Alemania & 0,1576 & 0,0630 & 0,1816 & 0,0870 & $-0,0588$ & 0,5250 \\
\hline Grecia & $-0,5142$ & 0,0000 & $-0,0089$ & 0,9520 & $-0,2438$ & 0,0290 \\
\hline Francia & 0,0409 & 0,6430 & 0,0294 & 0,7970 & 0,1837 & 0,0330 \\
\hline Irlanda & 0,0446 & 0,6260 & 0,1979 & 0,0920 & 0,0480 & 0,5860 \\
\hline Italia & $-0,1248$ & 0,1450 & 0,1122 & 0,4640 & $-0,1999$ & 0,0700 \\
\hline Luxemburgo & 0,2111 & 0,0100 & 0,3264 & 0,0020 & 0,1987 & 0,0160 \\
\hline
\end{tabular}




\section{CUADRO 3 (Continuación) \\ RESULTADOS DE LA ESTIMACIÓN DEL MODELO PROBIT MULTIVARIANTE}

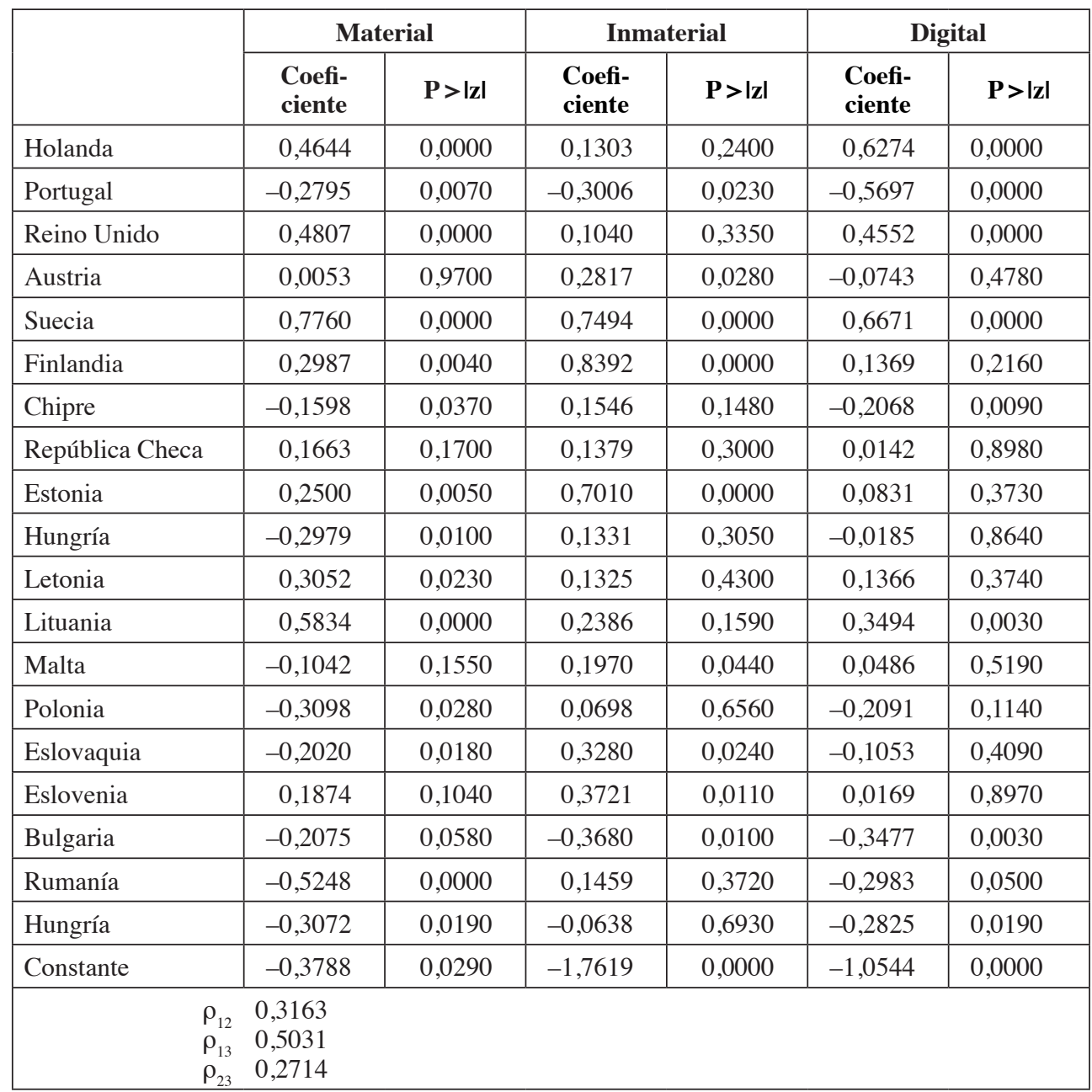

NOTA: Test de razón de verosimilitud de rho21 = rho31 = rho32 $=0$. Valor observado del estadístico de control $\operatorname{chi} 2(3)=2953,68 ;$ Prob $>$ chi2 $=0,0000$. N.$^{\circ}$ observ $=27881.246$ clusters: NUTS 2. Categorías omitidas de las variables categóricas en paréntesis.

FUENTE: Elaboración propia basada en datos del Eurobarómetro Especial 466 (2017).

La variable sexo es estadísticamente significativa para el acceso a patrimonio material e intangible, con un efecto negativo de ser hombre en línea con resultados anteriores que atribuyen las diferencias por sexo en la participación cultural a experiencias de socialización temprana. En el caso del coeficiente estimado para el acceso digital, este no es estadísticamente significativo. Esto se puede deber a la combinación del efecto negativo en las actividades complementarias y al efecto 
positivo relacionado con la evidencia existente para algunos de los países de nuestra muestra de una brecha digital por género que opera en contra de las mujeres. El ser extranjero residente en un país de la Unión Europea está asociado a una menor probabilidad de visitar instituciones patrimoniales y monumentos. Sin embargo, no encontramos evidencia de que exista un efecto de la nacionalidad de los individuos que esté relacionado con diferencias de socialización cultural entre los nacionales y los residentes no nacionales en los países de la Unión Europea para el patrimonio inmaterial ni para el acceso digital.

En nuestro análisis encontramos que la variable edad tiene efectos sobre la participación en las tres formas. Para patrimonio material encontramos efectos no monótonos, siendo los coeficientes positivos (respecto a la categoría base de 45 a 54 años) los de los grupos de edad 15-24, 35-44 y 65-74 años. La pertenencia al grupo de 75 años o mayores determina una menor probabilidad. En el caso del patrimonio intangible, la probabilidad aumenta para los de edad 55-64 y 65-74 años, probablemente relacionado con su mayor capacidad para haber adquirido conocimientos tradicionales. El acceso digital se reduce de forma significativa para los mayores de 65 años, evidenciando las diferencias de tasa de penetración y de uso digital entre el grupo de los mayores.

Respecto a la adscripción política manifestada por los individuos encontramos que, tanto para patrimonio material como inmaterial, los individuos que manifiestan posiciones más a la izquierda dentro de la escala tienen mayores probabilidades de participación. Se trata de un fenómeno monótono que no se da para los individuos que se posicionan más a la derecha. Esto podría ser interpretado en términos de la mayor apreciación del sustrato comunitario de los bienes del patrimonio cultural. En el caso del acceso digital, encontramos que tanto los que se posicionan a la derecha como a la izquierda tienen mayor probabilidad de participación, siendo el efecto no monótono.

La situación familiar tiene influencia en la participación a través de cada una de las tres formas consideradas. La presencia de niños en el hogar no tiene efecto estadísticamente significativo sobre ningún tipo de acceso.

Como es habitual en los modelos que determinan el efecto que tiene la educación sobre la participación cultural, el nivel de educación de los individuos tiene un efecto positivo y monótono para el acceso al patrimonio material. Este mismo efecto $-\mathrm{y}$ en magnitudes muy similares- es obtenido en nuestra estimación para el acceso digital. Sin embargo, para el caso de las manifestaciones del patrimonio intangible, no encontramos evidencia conclusiva de este efecto.

Encontramos también diferencias en función de la situación laboral y la ocupación de los individuos. Las más destacables operan en el ámbito del patrimonio material y del acceso digital. En concreto, dentro de esta última categoría parece que las ocupaciones más relacionadas con las habilidades digitales están relacionadas con una mayor participación. Por otra parte, para las visitas, encontramos que las ocupaciones relacionadas con un mayor estatus social también aumentan la probabilidad de participación por esta vía. 
Los recursos económicos del hogar también determinan la participación, aunque de forma diferente para cada una de las manifestaciones. Las dificultades económicas reportadas tienen un efecto monótono y estadísticamente significativo solo en el caso del acceso al patrimonio material. Sin embargo, la clase social declarada influye en las tres manifestaciones: de forma monótona para las visitas, de forma monótona solo hasta la clase media en el caso del patrimonio inmaterial, y de forma monótona para el acceso digital.

Por último, dentro de cualquiera de las tres manifestaciones estudiadas, hemos encontrado que, respecto a los hábitats rurales, las áreas urbanas grandes aumentan la probabilidad de participación. El efecto sobre el acceso digital es monótono y se encuentra un coeficiente positivo y estadísticamente significativo para las áreas urbanas pequeñas, pudiendo ser esto interpretado como evidencia adicional de la brecha digital entre el medio rural y urbano que existe aún en la Unión Europea.

Respecto a los efectos fijos de país, para lo que tomamos España como categoría base, se puede resaltar que los coeficientes estimados para cada una de las formas de participación tienen el mismo signo para cada uno de los países, salvo para Eslovaquia (con coeficiente estimado negativo para el patrimonio tangible y positivo para intangible).

\section{Conclusiones}

Los ciudadanos europeos acceden al patrimonio cultural por múltiples canales y parece existir una clara complementariedad entre ellos. Uno de los resultados que obtenemos es que el acceso de los ciudadanos al patrimonio cultural, cuando ampliamos el concepto, está mucho más extendido que cuando solo consideramos las meras visitas o la práctica de actividades relacionadas con las expresiones de la alta cultura o legítima, en la que aún perdura la distinción descrita por Bourdieu y que es explicada por el capital cultural individual cuando seguimos un análisis más económico. La práctica de manifestaciones relacionadas con el patrimonio inmaterial nos permite dibujar un panorama complementario al tradicional análisis de los hábitos de visitas.

El sector del patrimonio, sobre todo en lo que se refiere a bienes inmuebles y a instituciones, recibe una gran cantidad de recursos públicos para su conservación y gestión. Dada esa contribución pública, nos tendría que preocupar que no hubiera equidad en el acceso. Parece que hay factores que determinan que no se dé un acceso efectivo en iguales condiciones para algunos grupos sociales. Por ejemplo, en nuestro análisis hemos encontrado evidencia de las diferencias por clase social y por tamaño de población. Esta última diferencia es llamativa para el caso del patrimonio inmaterial, donde cabría pensar que las comunidades más pequeñas podrían tener más oportunidades de participar en tradiciones comunitarias. Parece que las variables que definen la brecha digital pueden determinar también un menor acceso digital al patrimonio y a los contenidos relacionados con él. Las grandes iniciativas 
de digitalización y de difusión del patrimonio cultural tendrían que tener en cuenta el reto adicional de llegar a esos ciudadanos de forma efectiva.

Como era de esperar, el acceso digital funciona como una actividad complementaria al acceso al patrimonio, pero la evidencia parece indicar que es más importante en el caso del patrimonio tangible (posiblemente relacionado también con otros hábitos de adquisición de información relacionados con el turismo cultural) que en el caso del patrimonio inmaterial. Además de atribuir parte de ese efecto a los hábitos digitales de la población, podemos pensar también en la relevancia de las variables de oferta. En general, existen muchos más recursos del patrimonio material inventariados, digitalizados y geocartografiados cuando los comparamos con proyectos de digitalización de manifestaciones de patrimonio inmaterial (como es el caso de la excelente iniciativa del Atlas del Patrimonio Inmaterial del Instituto Andaluz de Patrimonio Histórico).

En general, cabe esperar que las características que explican la mayor participación en cualquiera de las dimensiones serán también aquellas que permitan identificar a aquellos grupos de la población que estén más comprometidos con el patrimonio en términos de donaciones o de disposición para participar en mecanismos de gobernanza participativa (Comisión Europea, 2018). Quedan muchas preguntas abiertas que se podrían responder analizando la misma evidencia que aquí hemos presentado. Por ejemplo, sería interesante conocer más sobre el reconocimiento del patrimonio como recurso comunitario local o global o sobre visiones más o menos colectivas de responsabilidad sobre esos recursos comunes. Las respuestas a estas preguntas de investigación permitirían saber más sobre el posible papel del patrimonio cultural en la cohesión social que se busca en el ámbito europeo.

\section{Referencias bibliográficas}

Ateca-Amestoy, V. (2013). Demand for Cultural Heritage. En I. Rizzo y A. Mignosa (Eds.), Handbook on the Economics of Cultural Heritage (pp. 89-110). Cheltenham: Edward Elgar Publishing.

Ateca-Amestoy, V. (2018). Cultural Heritage Participation. Models for individual engagement and evidence for the EU. Economia della Cultura, 28 (4), 419-432.

Ateca-Amestoy, V., \& Prieto-Rodriguez, J. (2013). Forecasting accuracy of behavioural models for participation in the arts. European Journal of Operational Research, 229 (1), 124-131.

Ateca-Amestoy, V., \& Castiglione, C. (2014). Live and Digital Engagement with the visual arts. En 18th International Conference on Cultural Economics, Université du Québec (ESG-UQAM), Montreal, Canada.

Ateca-Amestoy, V., \& Castiglione, C. (2016). The consumption of cultural goods through the internet. How is it affected by the digital divide? (No AWP-04-2016). Association for Cultural Economics International.

Ateca-Amestoy, V., Gorostiaga, A., \& Rossi, M. (2019). Motivations and barriers to heritage engagement in Latin America: tangible and intangible dimensions. Journal of Cultural Economics, 1-27. https://doi.org/10.1007/s10824-019-09366-z 
Becker, G. S. (1965). A Theory of the Allocation of Time. The Economic Journal, 75 (299), 493-517.

Benhamou, F. (2011). Heritage. En R. Towse (Ed.), A Handbook of Cultural Economics, 2nd edition (pp. 229-235). Cheltenham: Edward Elgar Publishing.

Bonet, L. (2013). Heritage Tourism. En I. Rizzo \& A. Mignosa (Eds.), Handbook on the Economics of Cultural Heritage (pp. 386-401). Cheltenham: Edward Elgar Publishing.

Bourdieu, P. (1979). La distinction: Critique sociale du jugement. Paris: Les Éditions du Minuit.

Brida, J. G., Dalle Nogare, C., \& Scuderi, R. (2016). Frequency of museum attendance: motivation matters. Journal of Cultural Economics, 40(3), 261-283.

Cappellari, L., \& Jenkins, S. P. (2003). Multivariate probit regression using simulated maximum likelihood. The Stata Journal, 3(3), 278-294.

Comisión Europea (2017a). Mapping the Creative Value Chains. A study on the economy of culture in the digital age. https://doi.org/10.2766/868748

Comisión Europea (2017b). Eurobarometer 88.1. GESIS Datenarchiv, Köln. ZA6925 Datenfile Version 1.0.0 (2018). doi:10.4232/1.12959

Comisión Europea (2017c). Special Eurobarometer 466. October 2017. Cultural Heritage Report. https://doi.org/10.2766/576064

Comisión Europea (2018). Participatory governance of cultural heritage. Report of the Open Method of Coordination working group of Member States' experts. https://doi. org/10.2766/984757

Cheng, T. C., \& Wen, W. J. (2011). Determinants of performing arts attendance in Taiwan: a multivariate probit analysis. Applied Economics Letters, 18(15), 1437-1442.

De la Vega, P., Suarez-Fernández, S., Boto-García, D., \& Prieto-Rodriguez, J. (2019). Playing a play: online and live performing arts consumers profiles and the role of supply constraints. Journal of Cultural Economics, 1-26. https://doi.org/10.1007/s10824-01909367-y

Evrard, Y., \& Krebs, A. (2018). The authenticity of the museum experience in the digital age: the case of the Louvre. Journal of Cultural Economics, 42(3), 353-363.

Falk, M., \& Katz-Gerro, T. (2016). Cultural participation in Europe: Can we identify common determinants? Journal of Cultural Economics, 40(2), 127-162.

Greffe, X. (1989). La Valeur Économique du Patrimoine: la Demande et l'Offre de Monuments. Paris: Anthropos.

Guccio, C., Martorana, M. F., Mazza, I., \& Rizzo, I. (2016). Technology and Public Access to Cultural Heritage: The Italian Experience on ICT for Public Historical Archives. En K. J. Borowiecki, N. Forbers y A. Fresa (Eds.), Cultural Heritage in a Changing World (pp. 55-75). Springer, Cham.

Guccio, C., Lévi-Sacerdotti, S., \& Rizzo, I. (2017). An empirical investigation of cultural travellers' preferences and behaviours in a destination with mixed environmental features. En V. Ateca-Amestoy, V. Ginsburgh, I. Mazza, J. O’Hagan \& J. Prieto-Rodriguez (Eds.), Enhancing participation in the arts in the EU (pp. 249-265). Springer, Cham.

Lagaert, S., \& Roose, H. (2018). Gender and highbrow cultural participation in Europe: The effect of societal gender equality and development. International Journal of Comparative Sociology, 59(1), 44-68.

Lévy-Garboua, L. \& Montmarquette, C. (2011). Demand. En R. Towse (Ed.), A Handbook of Cultural Economics (2 ${ }^{\text {nd }}$ ed.). Cheltenham: Edward Elgar Publishing. 
Mihelj,S.,Leguina,A.,\& Downey,J.(2019).Cultural is digital: Cultural participation,diversity and the digital divide.New Media and Society.https://doi.org/10.1177/1461444818822816

Navarrete, T. (2013). Digital Cultural Heritage. En I. Rizzo \& A. Mignosa (Eds.), A Handbook on the Economics of Cultural Heritage (pp. 251-271). Cheltenham: Edward Elgar Publishing.

Noonan, D., \& Rizzo, I. (2017). Introducción al número especial sobre Turismo Cultural Economics of Cultural Tourism: Issues and Perspectives. Journal of Cultural Economics, 41(2), 95-10.

Novak-Leonard, J. L., \& Brown, A. S. (2011). Beyond Attendance: A Multi-Modal Understanding of Arts Participation. Based on the 2008 Survey of Public Participation in the Arts. Research Report\# 54. National Endowment for the Arts.

Palma, M. L., Palma, L., \& Aguado, L. F. (2013). Determinants of cultural and popular celebration attendance: the case study of Seville Spring Fiestas. Journal of Cultural Economics, 37(1), 87-107.

Peacock, A. (1995). The Political Economy of Heritage. Proceedings of the British Academy 87, 189-243.

Peacock, A. (1998). Does the Past have a Future? The Political Economy of Heritage. London: Institute of Economic Affairs.

Peacock, A., \&. Rizzo, I. (2008). The Heritage Game. Oxford: Oxford University Press.

Peterson, R. A., \& Kern, R. M. (1996). Changing highbrow taste: From snob to omnivore. American Sociological Review, 61(5), 900-907.

Reeves, A. (2019). How class identities shape highbrow consumption: A cross-national analysis of 30 European countries and regions. Poetics. https://doi.org/10.1016/j. poetic.2019.04.002

Rizzo, I., \& Towse, R. (Eds.) (2016). The Artful Economist: A New Look at Cultural Economics. Springer, Cham.

Sable, K. A., \& Kling, R. W. (2001). The double public good: A conceptual framework for 'shared experience' values associated with heritage conservation. Journal of Cultural Economics, 25(2), 77-89.

Seaman, B. A. (2005). Attendance and Public Participation in the Performing Arts: A Review of the Empirical Literature. Nonprofit Studies Program WP05-03, Georgia State University.

Suarez-Fernandez, S., Prieto-Rodriguez, J., \& Perez-Villadoniga, M. J. (2019). The changing role of education as we move from popular to highbrow culture. Journal of Cultural Economics. https://doi.org/10.1007/s10824-019-09355-2

Throsby, D. (1997). Seven Questions on the Economics of Cultural Heritage. En M. Hutter \& I. Rizzo (Eds.), Economic Perspectives on Cultural Heritage. London: Palgrave MacMillan.

Throsby, D. (2001). Economics and Culture. Cambridge: Cambridge University Press.

UNESCO (2009). Marco de Estadísticas Culturales UNESCO 2009. Montreal.

UNWTO (2018). Tourism and Culture Synergies. https://doi.org/10.18111/9789284418978

Vecco, M. (2010). A definition of cultural heritage: From the tangible to the intangible. Journal of Cultural Heritage, 11 (3), 321-324. 